\title{
Influence du mode de logement (sol/ cage) et de l'ascendance paternelle sur le comportement alimentaire et mérycique et la distribution de la taille des particules fécales chez les ovins
}

\author{
E Amouche, AG Deswysen \\ Université catholique de Louvain, faculté des sciences agronomiques, unité de génétique, \\ place Croix-du-Sud, 2, 1348, Louvain-la-Neuve, Belgique
}

La qualité de la mastication mérycique est très variable selon l'aliment, l'individu et le moment de la journée (Deswysen et al, 1989). L'objectif de cette étude est de préciser l'influence du lieu de logement et de l'ascendance paternelle sur le niveau d'ingestion volontaire et l'efficacité de mastication.

Douze moutons mâles castrés, de race Texel, âgés de $17-18$ mois $(53 \pm 5 \mathrm{~kg})$, et issus de 3 ascendances paternelles ( $A, B$ et C; $3 \times 4$ ) reçurent à volonté (à $9.00 \mathrm{~h}$ et $16.00 \mathrm{~h}$ ) un ensilage d'herbe préfanée en case individuelle au sol ( $35 \mathrm{j})$, puis en cages à métabolisme (15 j). Après une période préexpérimentale au sol (20 j), l'ingestion volontaire journalière fut mesurée durant les 2 périodes expérimentales successives au sol (15 j), puis en cages (15j). Le comportement alimentaire et mérycique fut enregistré en continu durant les 4 derniers jours de chaque période expérimentale. En même temps, lors du logement au sol, des fèces furent prélevées quotidiennement à $8 \mathrm{~h} 30$ au niveau du rectum, alors qu'en cages, les fèces émises durant les $24 \mathrm{~h}$ furent recueillies en totalité et échantillonnées. La distribution de la taille des particules fut mesurée par tamisage sous eau et exprimée en dimension moyenne pondérée.

L'ingestion volontaire journalière de matière sèche (IVMS) en cages à métabolisme est inférieure à celle au sol $\left(63,1\right.$ contre $71,0 \mathrm{~g}$ de $M S / k g P^{0,75}$; $P<0,05)$. Le comportement alimentaire et mérycique n'est pas significativement modifié par le lieu de logement. La dimension moyenne pondérée des particules fécales (DMPPF) est supérieure pour l'échantillon moyen des $24 \mathrm{~h}$ (en cages à métabolisme) comparé à l'échantillon rectal de $8 \mathrm{~h} 30$ (au sol) (190 contre $148 \mu \mathrm{m} ; P<0,01$ ), ce qui semble être en accord avec l'existence d'un rythme nycthéméral de la distribution de la taille des particules de digesta (Deswysen et al, 1989).

L'ascendance paternelle influence significativement les durées unitaires de rumination et de mastication (DUR et DUM), et la DMPPF. Les descendants de $A$ comparés à ceux de $B$ et de $C$ ingèrent légèrement plus $(69,7$ contre 66,5 et $64,9 \mathrm{~g}$ de $\mathrm{MS} / \mathrm{kg} \mathrm{P}^{0.75}$; NS), sans différence notable de digestibilité de la MS $(68,6$ contre 69,1 et $68,8 \%$; NS), tout en présentant des DUR inférieures $(6,5$ contre 7,6 et 8,3 min par $\mathrm{g}$ de $\left.M S / \mathrm{kg} \mathrm{P}^{0,75} ; P<0,001\right)$, ce qui se traduit par une DMPPF supérieure (195 contre 165 et $147 \mu \mathrm{m}$; $P<0,05)$.

En conclusion, le mode de logement influence significativement le niveau d'ingestion volontaire. L'efficacité de la mastication semble être sous dépendance génétique.

Deswysen AGT, Pond KR, Rivera-Villareal E, Ellis WC (1989) J Anim Sci 67, 1773-1783 